\title{
Association between promoter polymorphisms of the LIFR gene and schizophrenia with persecutory delusion in a Korean population
}

\author{
WON SUB KANG ${ }^{1 *}$, SU KANG KIM ${ }^{2 *}$, JIN KYUNG PARK ${ }^{1}$, AH RANG CHO ${ }^{1}$, \\ HAE JEONG PARK ${ }^{2}$, JOO-HO CHUNG ${ }^{2}$ and JONG WOO KIM ${ }^{1}$ \\ ${ }^{1}$ Department of Neuropsychiatry, School of Medicine, Kyung Hee University, Seoul 130-702; \\ ${ }^{2}$ Kohwang Medical Research Institute, School of Medicine, Kyung Hee University, \\ Seoul 130-701, Republic of Korea
}

Received July 11, 2011; Accepted October 3, 2011

DOI: $10.3892 / \mathrm{mmr} .2011 .618$

\begin{abstract}
Schizophrenia has been associated with an imbalance in inflammatory cytokines. Leukemia inhibitory factor receptor $\alpha$ (LIFR) is an integral component of the glycoprotein 130-LIFR signaling complex, which participates in signal transduction by members of the interleukin (IL)- 6 cytokine family. The aim of this study was to investigate whether genetic polymorphisms of the LIFR gene are associated with schizophrenia in the Korean population. We also explored possible associations between the polymorphisms and the clinical symptoms of schizophrenia. We selected three single nucleotide polymorphisms (SNPs) (rs2071237-200C/G, rs2071236 $-254 \mathrm{C} / \mathrm{T}$ and $\mathrm{rs} 6862038-962 \mathrm{~A} / \mathrm{G}$ ) on the promoter region of the LIFR gene and recruited 204 schizophrenia patients and 367 control subjects from the Korean population. All patients were evaluated according to the Operational Criteria Checklist for Psychotic Illness. SNP genotyping was performed by direct sequencing. An analysis of multiple logistic regression models (co-dominant 1 , co-dominant 2 , dominant, recessive and overdominant) was performed to evaluate odds ratios (ORs), 95\% confidence intervals (CIs) and p-values. No significant difference was revealed between schizophrenia patients
\end{abstract}

Correspondence to: Dr Jong Woo Kim, Department of Neuropsychiatry, School of Medicine, Kyung Hee University, 1 Hoegi-dong, Dongdaemun-gu, Seoul 130-702, Republic of Korea E-mail: psyjong@gmail.com

*Contributed equally

Abbreviations: LIFR, leukemia inhibitory factor receptor $\alpha$; LIF, leukemia inhibitory factor; IL, interleukin; gp130, glycoprotein 130; OPCRIT, Operation Criteria Checklist for Psychotic Illness; SNP, single nucleotide polymorphism; HWE, Hardy-Weinberg equilibrium; LD, linkage disequilibrium

Key words: leukemia inhibitory factor receptor $\alpha$, cytokine, persecutory delusion, schizophrenia, single nucleotide polymorphism and controls. However, a significant association was detected between the LIFR gene polymorphisms and schizophrenia patients with persecutory delusion ( $\mathrm{rs} 2071236, \mathrm{OR}=2.18,95 \%$ $\mathrm{CI}=1.20-3.94, \mathrm{P}=0.009$ in the overdominant model; rs6862038, $\mathrm{OR}=2.08,95 \% \mathrm{CI}=1.16-3.74, \mathrm{P}=0.013$ in the overdominant model). The present study suggests that the LIFR gene may be related to schizophrenia with persecutory delusion in the Korean population.

\section{Introduction}

Accumulating evidence indicates that schizophrenia is associated with an imbalance in T helper-1/T helper-2 (Th1/Th2) cytokines, with a shift towards the Th2 system (1), and that cytokines contribute to the pathogenesis of schizophrenia $(2,3)$. Indeed, previous studies have revealed that the levels of interleukin-1 receptor antagonist (IL-1RA), soluble interleukin-2 receptor (IL-2R) and interleukin-6 (IL-6) were increased, and that the level of IL-2 secretion was decreased in schizophrenia patients (4). In addition, genetic studies revealed the association of polymorphisms and/or haplotypes of cytokine genes including IL-10 (5,6), IL-6 (6) and the IL-1 gene complex (IL-1 $\alpha$, IL-1 $\beta$ and IL-1RA) with schizophrenia (7).

Leukemia inhibitory factor (LIF) is a member of the IL-6 cytokine family comprising IL-6, oncostatin M, IL-11, ciliary neurotropic factor and cardiotropin-1; it signals via binding to a heterodimeric glycoprotein 130 (gp130)/LIF receptor (LIFR) complex (8). In the central nerve system, LIF was found to regulate the neuronal phenotype and coordinate astrocyte, oligodendrocyte, microglia and inflammatory cell responses through binding with the gp130/LIFR complex (8-10). In a previous study, single nucleotide polymorphisms (SNPs) and haplotypes of the LIF gene were found to be associated with schizophrenia and with clinical phenotypes of schizophrenia, suggesting the LIF gene as a candidate gene of schizophrenia (11). Moreover, in an experimental study, LIF-knockout mice displayed abnormal prepulse inhibition in the acoustic startle test during and following adolescence, which was considered as one of the intermediate phenotypes observed in schizophrenia patients (12). 
In light of these findings, we speculated that LIF signaling may play a role in the pathophysiology of schizophrenia. In particular, considering that LIF signaling is mainly mediated through its receptor, LIFR may be related to schizophrenia. The LIFR gene is structured by six different domains encoding for a 1,097-amino acid transmembrane protein. The LIFR gene maps to the short arm of chromosome 5 in the p13-12 region (13). In this study, we investigated the genetic associations between schizophrenia and the LIFR gene in the Korean population through an analysis of the promoter SNPs of the LIFR gene.

\section{Materials and methods}

Subjects and clinical assessment. All participants were ethnically Korean and unrelated to each other. A total of 204 Korean schizophrenia patients [mean age \pm standard deviation (SD), $41.8 \pm 10.8$ years] and 367 healthy control individuals (44.3 \pm 6.3 years) were evaluated. All patients were diagnosed with schizophrenia by well-trained psychiatrists according to the Diagnostic and Statistical Manual of Mental Disorders (4th edition) and were assessed according to the Operational Criteria (OPCRIT) checklist (14). The core and most common symptoms of schizophrenia are hallucinations and delusions. The most common delusions in schizophrenia patients are persecutory delusions, which are experienced by up to $80 \%$ of inpatients. Thus, we selected the persecutory delusion and hallucination criteria from the OPCRIT checklist to further analyze the association between the clinical symptoms of schizophrenia and the LIFR gene. For analysis by clinical symptoms, in particular, patients with item 54 on the OPCRIT checklist were classified as the persecutory delusion group and patients with any of the items 73-77 formed the hallucination group (15). In the present genotyped sample of schizophrenia patients, participants were subdivided into those who experienced persecutory delusion $(n=124,60.8 \%)$ and those who did not $(n=80,39.2 \%)$; and those who experienced hallucinations $(n=101,49.5 \%)$ and those who did not $(n=103,50.5 \%)$. The control group was recruited from subjects visiting the hospital for routine health checkups. Control subjects had no psychiatric disorders or history thereof. Written informed consent was obtained from all subjects involved in the study, and the study was approved by the Ethics Review Committee of the Medical Research Institute, Kyung Hee University Medical Center, Seoul, Korea.

SNP selection and genotyping. SNPs for the LIFR gene were selected from the promoter regions within $-1000 \mathrm{bp}$ of the transcriptional start sites, using HapMap (www.hapmap.org/; genome build 36) and SNP databases (dbSNP) (www.ncbi. nlm.nih.gov/SNP; build 132). The SNPs without genotype frequency data for the Asian population, and with a heterozygosity $\leq 0.05$ or a minor allele frequency $\leq 0.05$ were excluded. Finally, we selected three promoter SNPs as follows: rs2071237 (-200C/G), rs2071236 (-254C/T) and rs6862038 (-962A/G).

A DNA isolation kit for blood (Roche, Indianapolis, USA) was used to isolate DNA from a peripheral blood sample. SNP genotyping was conducted by direct sequencing using the following primers for each SNP: rs2071237 (sense, 5'-CCTTGAACCACAATCCAAGACAC-3' and antisense, 5'-GAAAGTCTGGGTAAGTACAGGTC-3'); rs2071236 (sense, 5'-GGATCACGAGGTCAGGAGATCGAG-3' and antisense, 5'-GTGCAGTTCTGCAGAGCGAAAGGG-3'); rs6862038 (sense, 5'-GTAAGGGCGATTTTACACTGTCCG-3' and antisense, 5'-ATTCTTAGCCACGGAGGGAGCCTC-3'). The PCR products were sequenced using an ABI PRISM 3730XL analyzer (PE Applied Biosystems, Foster City, CA, USA). Sequence data were analyzed using SeqManII software (DNAStar Inc., Madison, WI, USA).

Statistical analysis. For the analysis of Hardy-Weinberg equilibrium (HWE) and genotype frequencies, SNPAnalyzer Pro (Istech Inc., Goyang, Korea) and SNPStats (http://bioinfo. iconcologia.net/index.php) were used. The associations between genotypes of SNPs and schizophrenia, as well as any associations between SNPs and schizophrenia subgroups were estimated by computing the odds ratios (ORs) and their 95\% confidence intervals (CIs) with logistic regression analyses, controlling for age and gender as covariables. In the logistic regression analysis for each SNP, co-dominant 1 (major allele homozygotes versus heterozygotes), co-dominant 2 (major allele homozygotes versus minor allele homozygotes), dominant (major allele homozygotes versus minor allele homozygotes plus heterozygotes), recessive (major allele homozygotes plus heterozygotes versus minor allele homozygotes) or overdominant (heterozygotes versus homozygotes) models were used. To avoid chance findings due to multiple testing, the Bonferroni correction was applied by lowering the significance levels to $\mathrm{p}=0.016(\mathrm{p}=0.05 / 3)$ for the three SNPs.

\section{Results}

Genetic association of LIFR SNPs with schizophrenia patients. When the association between the promoter SNPs of the LIFR gene and schizophrenia was analyzed, differences in the genotype frequencies of one SNP were found between the schizophrenia patients and the controls (rs2071237, OR=0.66, 95\% $\mathrm{CI}=0.45-0.97, \mathrm{P}=0.027$ in the co-dominant 1 model; $\mathrm{OR}=0.65,95 \% \mathrm{CI}=0.45-0.94, \mathrm{P}=0.023$ in the dominant model; Table I). The $\mathrm{C}$ allele frequency of rs2071237 was higher in the schizophrenia group than in the control group $(\mathrm{OR}=1.29$, 95\% $\mathrm{CI}=1.01-1.65, \mathrm{P}=0.045$; data not shown). However, these associations were not significant after applying the Bonferroni correction for multiple testing (Table I).

To further analyze the haplotype structure in our samples, we identified a linkage disequilibrium (LD) block between the three SNPs in the control subjects, using pairwise D' values (D'=1) by Gabriel's criteria (16). Three SNPs (rs2071237, rs2071236 and rs6862038) of the LIFR gene were analyzed for linkage disequilibrium (LD) and haplotypes using Haploview 4.2. The LD block was composed of rs2071237, rs2071236 and rs6862038 (data not shown). There were four haplotypes in the LD block (haplotype CCG, frequency=0.407; GTA, frequency $=0.313$; GCG, frequency $=0.216$; and GCA, frequency $=0.063$; Table II). Haplotype GCG in the LD block was found to be associated with schizophrenia (Chi square $=4.468, \mathrm{P}=0.0345$; Table II). However, this association disappeared following the Bonferroni correction (Table II).

Clinical phenotypes in schizophrenia patients. An association between genotype and specific clinical symptoms 
Table I. Genotype frequencies of promoter polymorphisms of LIFR in patients with schizophrenia and control subjects ${ }^{\mathrm{a}}$.

\begin{tabular}{|c|c|c|c|c|c|c|c|c|}
\hline \multirow{2}{*}{$\begin{array}{l}\text { SNP } \\
\text { location }\end{array}$} & \multirow[t]{2}{*}{ Genotype } & \multicolumn{2}{|c|}{ Schizophrenia } & \multicolumn{2}{|c|}{ Control } & \multirow[t]{2}{*}{ Model } & \multirow[t]{2}{*}{ OR $(95 \%$ CI $)$} & \multirow[t]{2}{*}{ P-value } \\
\hline & & $\mathrm{n}$ & $\%$ & $\mathrm{n}$ & $\%$ & & & \\
\hline \multirow{5}{*}{$\begin{array}{l}\text { rs2071237 } \\
-200\end{array}$} & $\mathrm{G} / \mathrm{G}$ & 80 & 39.4 & 107 & 29.5 & Co-dominant 1 & $0.66(0.45-0.97)$ & 0.027 \\
\hline & $\mathrm{C} / \mathrm{G}$ & 97 & 47.8 & 199 & 54.8 & Co-dominant 2 & $0.63(0.36-1.09)$ & 0.077 \\
\hline & $\mathrm{C} / \mathrm{C}$ & 26 & 12.8 & 57 & 15.7 & Dominant & $0.65(0.45-0.94)$ & 0.023 \\
\hline & & & & & & Recessive & $0.80(0.48-1.33)$ & 0.390 \\
\hline & & & & & & Overdominant & $0.76(0.54-1.08)$ & 0.120 \\
\hline \multirow{5}{*}{$\begin{array}{l}\text { rs2071236 } \\
-254\end{array}$} & $\mathrm{C} / \mathrm{C}$ & 97 & 47.8 & 160 & 44.1 & Co-dominant 1 & $0.78(0.54-1.12)$ & 0.229 \\
\hline & $\mathrm{C} / \mathrm{T}$ & 86 & 42.4 & 177 & 48.8 & Co-dominant 2 & $1.24(0.65-2.37)$ & 0.463 \\
\hline & $\mathrm{T} / \mathrm{T}$ & 20 & 9.8 & 26 & 7.2 & Dominant & $0.84(0.59-1.19)$ & 0.320 \\
\hline & & & & & & Recessive & $1.41(0.76-2.62)$ & 0.280 \\
\hline & & & & & & Overdominant & $0.75(0.53-1.07)$ & 0.110 \\
\hline \multirow{5}{*}{$\begin{array}{l}\text { rs6862038 } \\
-962\end{array}$} & $\mathrm{G} / \mathrm{G}$ & 79 & 38.9 & 133 & 36.6 & Co-dominant 1 & $0.79(0.54-1.15)$ & 0.337 \\
\hline & $\mathrm{A} / \mathrm{G}$ & 93 & 45.8 & 188 & 51.8 & Co-dominant 2 & $1.19(0.69-2.06)$ & 0.431 \\
\hline & $\mathrm{A} / \mathrm{A}$ & 31 & 15.3 & 42 & 11.6 & Dominant & $0.86(0.60-1.24)$ & 0.420 \\
\hline & & & & & & Recessive & $1.36(0.82-2.26)$ & 0.240 \\
\hline & & & & & & Overdominant & $0.75(0.53-1.07)$ & 0.110 \\
\hline
\end{tabular}

${ }^{a}$ To avoid chance findings due to multiple testing, $\mathrm{P}<0.016$ was considered statistically significant. SNP, single nucleotide polymorphism; OR, odds ratio; $\mathrm{CI}$, confidence interval; $\mathrm{n}$, number of subjects.

Table II. Frequencies of haplotypes in schizophrenia and control subjects ${ }^{\mathrm{a}}$.

\begin{tabular}{|c|c|c|c|c|c|c|c|}
\hline \multirow[t]{2}{*}{ Haplotype } & \multirow[t]{2}{*}{ Frequency } & \multicolumn{2}{|c|}{ Schizophrenia } & \multicolumn{2}{|c|}{ Control } & \multirow[t]{2}{*}{ Chi square } & \multirow[t]{2}{*}{$\mathrm{P}$-value } \\
\hline & & + & - & + & - & & \\
\hline $\mathrm{CCG}$ & 0.407 & 150.9 & 257.1 & 309.9 & 414.1 & 3.641 & 0.0564 \\
\hline GTA & 0.313 & 126.0 & 282.0 & 228.0 & 496.0 & 0.045 & 0.8319 \\
\hline GCG & 0.216 & 102.1 & 305.9 & 142.1 & 581.9 & 4.468 & 0.0345 \\
\hline GCA & 0.063 & 28.9 & 379.1 & 42.9 & 681.1 & 0.604 & 0.4369 \\
\hline
\end{tabular}

${ }^{a}$ To avoid chance findings due to multiple testing, $\mathrm{P}<0.016$ was considered statistically significant. Haplotypes are comprised of rs 2071237 , rs2071236 and rs6862038.

of schizophrenia patients was elucidated. We also assessed the association of the three SNPs of the LIFR gene with the symptoms of persecutory delusion and hallucinations in the schizophrenia patients. Differences were found between those with and without symptoms of persecutory delusion (rs2071236, $\mathrm{OR}=2.06,95 \% \mathrm{CI}=1.11-3.81, \mathrm{P}=0.024$ in the co-dominant 1 model, $\mathrm{OR}=2.18,95 \% \mathrm{CI}=1.20-3.94, \mathrm{P}=0.009$ in the overdominant model; rs6862038, OR=2.06, 95\% CI=1.09-3.87, $\mathrm{P}=0.025$ in the co-dominant 1 model, $\mathrm{OR}=2.08,95 \% \mathrm{CI}=1.16-3.74$, $\mathrm{P}=0.013$ in the overdominant model; Table III). Following the Bonferroni correction, the significant association remained (rs2071236, $\mathrm{P}=0.009$; rs6862038, $\mathrm{P}=0.013$; Table III). The distribution of genotype and allele frequencies did not differ significantly between schizophrenia patients with and without hallucinations (data not shown). No haplotype association was found between the selected SNPs and hallucination criteria or persecutory delusion in the schizophrenia patient group (data not shown).

\section{Discussion}

In the present study, we explored the possibility of a genetic association between the LIFR gene and schizophrenia in the Korean population. However, no significant correlation was found between schizophrenia and the promoter region SNPs of the LIFR gene. An additional analysis was performed to explore the correlation between the three SNPs and clinical symptoms of schizophrenia, such as persecutory delusion and hallucinations. The results demonstrated that rs2071236 and rs6862038 were associated with persecutory delusion.

Previously, a proteomic study reported that the levels of glial fibrillary acidic protein (GFAP) expression, which is a cytoskeletal marker of astroglia, was reduced in the brains of individuals with schizophrenia (17). Another group demonstrated that the GFAP mRNA levels in the white matter of the anterior cingulate cortex were decreased in schizophrenia patients (18). These results indicate that astrocytes play a 
Table III. Genotype frequencies of promoter polymorphisms of LIFR in schizophrenia patients with and without persecutory delusiona .

\begin{tabular}{|c|c|c|c|c|c|c|c|c|}
\hline \multirow{3}{*}{$\begin{array}{l}\text { SNP } \\
\text { location }\end{array}$} & \multirow{3}{*}{ Genotype } & \multicolumn{4}{|c|}{ Persecutory delusion } & \multirow{3}{*}{ Model } & \multirow{3}{*}{ OR $(95 \% \mathrm{CI})$} & \multirow{3}{*}{ P-value } \\
\hline & & \multicolumn{2}{|c|}{ With } & \multicolumn{2}{|c|}{ Without } & & & \\
\hline & & $\mathrm{n}$ & $\%$ & $\mathrm{n}$ & $\%$ & & & \\
\hline \multirow{5}{*}{$\begin{array}{l}\text { rs2071237 } \\
-200\end{array}$} & $\mathrm{G} / \mathrm{G}$ & 53 & 43.1 & 27 & 33.8 & Co-dominant 1 & $0.74(0.40-1.37)$ & 0.377 \\
\hline & $\mathrm{C} / \mathrm{G}$ & 58 & 47.1 & 39 & 48.8 & Co-dominant 2 & $0.44(0.18-1.08)$ & 0.071 \\
\hline & $\mathrm{C} / \mathrm{C}$ & 12 & 9.8 & 14 & 17.5 & Dominant & $0.66(0.37-1.19)$ & 0.160 \\
\hline & & & & & & Recessive & $0.52(0.23-1.19)$ & 0.120 \\
\hline & & & & & & Overdominant & $0.91(0.52-1.61)$ & 0.760 \\
\hline \multirow{5}{*}{$\begin{array}{l}\text { rs2071236 } \\
-254\end{array}$} & $\mathrm{C} / \mathrm{C}$ & 53 & 43.1 & 44 & 55.0 & Co-dominant 1 & $2.06(1.11-3.81)$ & 0.024 \\
\hline & $\mathrm{C} / \mathrm{T}$ & 61 & 49.6 & 25 & 31.2 & Co-dominant 2 & $0.72(0.27-1.91)$ & 0.433 \\
\hline & $\mathrm{T} / \mathrm{T}$ & 9 & 7.3 & 11 & 13.8 & Dominant & $1.66(0.94-2.94)$ & 0.082 \\
\hline & & & & & & Recessive & $0.52(0.20-1.32)$ & 0.170 \\
\hline & & & & & & Overdominant & $2.18(1.20-3.94)$ & 0.009 \\
\hline rs6862038 & $\mathrm{G} / \mathrm{G}$ & 42 & 34.1 & 37 & 46.2 & Co-dominant 1 & $2.06(1.09-3.87)$ & 0.025 \\
\hline \multirow[t]{4}{*}{-962} & $\mathrm{~A} / \mathrm{G}$ & 65 & 52.9 & 28 & 35.0 & Co-dominant 2 & $0.96(0.42-2.21)$ & 0.883 \\
\hline & $\mathrm{A} / \mathrm{A}$ & 16 & 13.0 & 15 & 18.8 & Dominant & $1.67(0.94-3.00)$ & 0.082 \\
\hline & & & & & & Recessive & $0.66(0.30-1.42)$ & 0.290 \\
\hline & & & & & & Overdominant & $2.08(1.16-3.74)$ & 0.013 \\
\hline
\end{tabular}

${ }^{a}$ To avoid chance findings due to multiple testing, $\mathrm{P}<0.016$ was considered statistically significant. Bold characters indicate statistical significance. SNP, single nucleotide polymorphism; OR, odds ratio; CI, confidence interval; n, number of subjects.

significant role in the pathogenesis of schizophrenia. The functional implication of LIFR in neuronal and glial development has been studied both in vivo and in vitro (19). In addition, it has been established that LIFR is also implicated in the development of astrocytes $(20,21)$. These studies revealed that LIFR mutant embryos completely lack GFAP expression in the spinal cord and hindbrain (21). In addition to the role of LIFR in the development of astrocytes, serum LIFR concentrations were significantly increased after starting treatment with antipsychotics in schizophrenia patients (22). Moreover, in our study, the LIFR gene was associated with persecutory delusion in schizophrenia. Taken together, LIFR may be implicated in the pathogenesis of schizophrenia.

To find whether the alleles of rs2071236 and rs6862038 relate to transcription factors, we used the online program 'AliBaba 2.1' (http://www.gene-regulation.com/pub/programs/ alibaba2). At the rs2071236 SNP site, C-containing sequences are shown to act with ER and CREBP1 transcription factors, but CREBP1 disappears in T-containing sequences. At the rs2071236 SNP site, the A-containing sequence binds with HNF-1 and FTZ, and T-containing sequences do not bind with any transcription factor. Therefore, this SNP may regulate gene expression by controlling the binding of transcription factors.

For a clear understanding of the pathogenesis of schizophrenia, further research is required to investigate the corellation between other SNPs of the LIFR gene and schizophrenia. In addition, replication studies using larger samples need to be performed in several populations to confirm our initial findings. Nevertheless, even with these limitations, our results suggest the possibility that polymorphisms of the LIFR gene may be related to persecutory delusion in schizophrenia.

In conclusion, a significant association was revealed between LIFR gene polymorphisms and symptoms of persecutory delusion in schizophrenia patients. In particular, rs2071236 and rs6862038 were associated with persecutory delusion. These results suggest that the LIFR gene may be related to the susceptibility to persecutory delusion in schizophrenia.

\section{Acknowledgements}

This study was supported by a grant from the Kyung Hee University (KHU-20090641).

\section{References}

1. Muller N and Schwarz M: Schizophrenia as an inflammationmediated dysbalance of glutamatergic neurotransmission. Neurotox Res 10: 131-148, 2006.

2. Nawa H, Takahashi M and Patterson PH: Cytokine and growth factor involvement in schizophrenia - support for the developmental model. Mol Psychiatry 5: 594-603, 2000.

3. Nawa $\mathrm{H}$ and Takei $\mathrm{N}$ : Recent progress in animal modeling of immune inflammatory processes in schizophrenia: implication of specific cytokines. Neurosci Res 56: 2-13, 2006.

4. Potvin S, Stip E, Sepehry AA, Gendron A, Bah R and Kouassi E: Inflammatory cytokine alterations in schizophrenia: a systematic quantitative review. Biol Psychiatry 63: 801-808, 2008.

5. Lung FW, Yang MC and Shu BC: The interleukin 10 promoter haplotype ACA and long-form variant of the DRD4 uVNTR polymorphism are associated with vulnerability to schizophrenia. Psychiatry Res 188: 294-296, 2011. 
6. Paul-Samojedny M, Kowalczyk M, Suchanek R, et al: Functional polymorphism in the interleukin- 6 and interleukin-10 genes in patients with paranoid schizophrenia - a case control study. J Mol Neurosci 42: 112-119, 2010.

7. Xu M and He L: Convergent evidence shows a positive association of interleukin-1 gene complex locus with susceptibility to schizophrenia in the Caucasian population. Schizophr Res 120: 131-142, 2010.

8. Taga T and Kishimoto T: Gp130 and the interleukin-6 family of cytokines. Annu Rev Immunol 15: 797-819, 1997.

9. He F, Ge W, Martinowich K, et al: A positive autoregulatory loop of Jak-STAT signaling controls the onset of astrogliogenesis. Nat Neurosci 8: 616-625, 2005.

10. Koblar SA, Turnley AM, Classon BJ, et al: Neural precursor differentiation into astrocytes requires signaling through the leukemia inhibitory factor receptor. Proc Natl Acad Sci USA 95: 3178-3181, 1998.

11. Okahisa Y, Ujike H, Kunugi H, et al: Leukemia inhibitory factor gene is associated with schizophrenia and working memory function. Prog Neuropsychopharmacol Biol Psychiatry 34: $172-176,2010$.

12. Watanabe Y, Hashimoto S, Kakita A, et al: Neonatal impact of leukemia inhibitory factor on neurobehavioral development in rats. Neurosci Res 48: 345-353, 2004.

13. Gearing DP, Druck T, Huebner K, et al: The leukemia inhibitory factor receptor (LIFR) gene is located within a cluster of cytokine receptor loci on mouse chromosome 15 and human chromosome 5p12-p13. Genomics 18: 148-150, 1993.

14. McGuffin P, Farmer A and Harvey I: A polydiagnostic application of operational criteria in studies of psychotic illness Development and reliability of the OPCRIT system. Arch Gen Psychiatry 48: 764-770, 1991.
15. Fanous AH, van den Oord EJ, Riley BP, et al: Relationship between a high-risk haplotype in the DTNBP1 (dysbindin) gene and clinical features of schizophrenia. Am J Psychiatry 162: 1824-1832, 2005.

16. Gabriel SB, Schaffner SF, Nguyen H, et al: The structure of haplotype blocks in the human genome. Science 296: 2225-2229, 2002.

17. Johnston-Wilson NL, Sims CD, Hofmann J-P, et al: Diseasespecific alterations in frontal cortex brain proteins in schizophrenia, bipolar disorder, and major depressive disorder. Mol Psychiatry 5: 142-149, 2005.

18. Webster MJ, O'Grady J, Kleinman JE and Weickert CS: Glial fibrillary acidic protein mRNA levels in the cingulate cortex of individuals with depression, bipolar disorder and schizophrenia. Neuroscience 133: 453-461, 2005.

19. Li M, Sendtner M and Smith A: Essential function of LIF receptor in motor neurons. Nature 378: 724-727, 1995.

20. Barnabé-Heider F, Wasylnka JA, Fernandes KJ, et al: Evidence that embryonic neurons regulate the onset of cortical gliogenesis via cardiotrophin-1. Neuron 48: 253-265, 2005.

21. Ware CB, Horowitz MC, Renshaw BR, et al: Targeted disruption of the low-affinity leukemia inhibitory factor receptor gene causes placental, skeletal, neural and metabolic defects and results in perinatal death. Development 121: 1283-1299, 1995.

22. Maes M, Bocchio Chiavetto L, Bignotti S, et al: Increased serum interleukin- 8 and interleukin-10 in schizophrenic patients resistant to treatment with neuroleptics and the stimulatory effects of clozapine on serum leukemia inhibitory factor receptor. Schizophr Res 54; 281-291, 2002. 